\title{
Uncomplicated Plasmodium vivax malaria in pregnancy associated with mortality from acute respiratory distress syndrome
}

Rose McGready ${ }^{1,2,3^{*}}$, Klanarong Wongsaen ${ }^{1}$, Cindy S Chu', Nay Win Tun ${ }^{1}$, Kesinee Chotivanich², Nicholas J White ${ }^{2,3}$ and François Nosten ${ }^{1,2,3}$

\begin{abstract}
The association between severe malaria and Plasmodium vivax species is contentious. On the Thai-Myanmar border, all pregnant women are followed systematically with active weekly malaria screening. Over a 27-year period of providing antenatal care, 48,983 have been prospectively followed until pregnancy outcome (miscarriage or delivery) and 4,298 women have had $P$. vivax detected at least once. Reported here is the first known $P$. vivax-associated death amongst these women. The initial patient presentation was of uncomplicated $P$. vivax ( $0.5 \%$ parasitaemia) in a term, multigravida woman who responded rapidly to oral artesunate and mefloquine treatment, clearing her blood stage parasites within 48 hours. The patient appeared well, was ambulatory and due to be discharged but became unwell with acute respiratory distress syndrome (ARDS) requiring ventilation three days (67 hours) into treatment. Despite induction and delivery of a stillborn foetus, ventilatory requirements increased and the patient died on day 7 . The patient had a low body mass index. Sensitive detection with nested PCR confirmed only the presence of $P$. vivax species and concomitant infections such as tuberculosis and human immunodeficiency virus (HIV) were also ruled out. The contemporaneous treatment of acute uncomplicated $P$. vivax and the onset of ARDS on day 3 in this patient implies a possible but unconfirmed association with death in this patient. Assuming this death was caused by $P$. vivax, the risk of ARDS-related maternal mortality in this setting did not differ significantly between Plasmodium falciparum and $P$. vivax $(0.24$ per 1,000 $(1 / 4,158)$ versus 0.23 per $1,000(1 / 4,298)$, contrary to the increased risk of maternal mortality from $P$. falciparum compared to $P$. vivax, 2.89 per $1,000(12 / 4,158)$ versus 0.23 per $1,000(1 / 4,298), P=0.003$.
\end{abstract}

Keywords: Acute respiratory distress syndrome, Maternal mortality, Plasmodium vivax, Severe malaria

\section{Background}

On the Thai-Myanmar border Plasmodium falciparum [1] and Plasmodium vivax [2] malaria are associated with adverse outcomes in pregnancy, with $P$. falciparum species typically involved in mortality reports [3]. Tests of lung function in both uncomplicated $P$. falciparum and $P$. vivax malaria have demonstrated a decrease in diffusion capacity which has been attributed to a reduction in the pulmonary capillary vascular component of gas transfer [4]. Following treatment, gas transfer is progressively reduced due to

\footnotetext{
* Correspondence: rose@shoklo-unit.com

'Shoklo Malaria Research Unit, Mahidol-Oxford Tropical Medicine Research Unit, Faculty of Tropical Medicine, Mahidol University, Mae Sot, Thailand ${ }^{2}$ Mahidol-Oxford Tropical Medicine Research Unit, Faculty of Tropical Medicine, Mahidol University, Bangkok, Thailand

Full list of author information is available at the end of the article
}

interstitial and alveolar oedema and is more prominent in $P$. vivax compared with $P$. falciparum infection. Sequestration of $P$. vivax in human lung tissue has not yet been shown, suggesting non-sequestering mechanisms for acute respiratory distress syndrome (ARDS), such as soluble mediators causing endothelial damage, hypoxia and systemic shock, resulting in diffuse alveolar-capillary damage $[5,6]$. Reported in this manuscript is a case of ARDS commencing on day 3 into treatment for uncomplicated $P$. vivax infection when the patient appeared to be in the recovery phase of the illness.

\section{Case presentation}

In this case a 26-year old, gravida 3, parity 2, ethnic Mon pregnant woman from Myanmar first presented to the Shoklo Malaria Research Unit (SMRU) antenatal clinic 
(ANC) at 30 weeks and six days of pregnancy in early February 2013. This patient was a literate, non-smoker, with no history of prior medical problems whose prior obstetric history included one normal vaginal delivery and the most recent delivery was a caesarean section in Moulmein (Mawlamyine) for breech presentation. She was a migrant worker (migrating between Myanmar and Thailand) and resident for one month at her present address. At the first ANC visit, her vital signs and physical examination were normal except her body weight, which was low at $34 \mathrm{~kg}$ (BMI only $17.1 \mathrm{~kg} / \mathrm{m}^{2}$ in late pregnancy). Her screening tests were negative for malaria, HIV, syphilis, and hepatitis B; and glucose-6-phosphate dehydrogenase status was normal. Her haematocrit (HCT) was low at $26 \%$ and haemoglobin typing confirmed she had $\beta$-thalassaemia trait, so ferrous and folic acid supplements were commenced. At routine weekly ANC consultations her malaria smear screening was negative a total of three times. Note in this area of the border with multidrug-resistant $P$. falciparum and $P$. vivax [7], women are actively and frequently screened by malaria smear (weekly and 24-hour service) and all positive cases are treated (regardless of symptoms) as there is no drug with proven safety and efficacy for prophylaxis during pregnancy.

At a gestation of 36 weeks and six days, six weeks (day 0) after booking, the patient complained of a seven-day history of fever, along with muscle, joint and abdominal pain, dizziness, palpitations, anorexia, and sleep disturbance. On examination the body temperature was slightly elevated at $37.7^{\circ} \mathrm{C}$, blood pressure $100 / 65 \mathrm{mmHg}$, respiratory rate 26 breaths per minute (similar to six weeks earlier when it was 24 breaths per minute), pulse rate 90 per minute and the fetal heart rate was 134 beats per minute. Malaria smear showed a $0.5 \%(16,238 / \mathrm{uL}) P$. vivax parasitaemia with all stages of the parasite present (Table 1 ). Her complete blood count showed a haemoglobin of $8.4 \mathrm{mg} /$ $\mathrm{dL}$, HCT 26.7\%, mean cell volume $60.8 \mathrm{fl}$, white blood cells $1.5 \times 10^{3} / \mathrm{ml}$ with differential counts $\left(\times 10^{3} / \mathrm{ml}\right)$ of neutrophils (1.1), lymphocytes (0.3), monocytes (0.1), eosinophils (0) and basophils (0), and platelet count $138 \times$ $10^{3} / \mu \mathrm{L}$ and C-reactive protein was $51.2 \mathrm{mg} / \mathrm{L}$, consistent with a diagnosis of acute $P$. vivax. Microbiological examination of blood and urine, and scrub typhus IgG serology were negative. Treatment with fixed mefloquine and artesunate (Far-Manguinhos, Brazil) (artesunate/mefloquine $100 \mathrm{mg} / 220 \mathrm{mg}$ ) was started at one tablet once daily for three days with a good clinical response. This treatment was used in place of three days of chloroquine, the standard treatment, as the patient agreed to participate in a clinical trial [8]. Three supervised anti-malarial treatment doses were completed by 48 hours. The parasite count reduced rapidly (Table 1 ). The fever clearance time was 12 hours and the clinical symptoms reduced on day 1 with the patient only complaining of anorexia but less than on the previous day, and on day 2 anorexia (again less than the previous day) and new onset dizziness and palpitations.

On day 2, the patient was well and she was due to be discharged the following day. Around midnight on day 2 she complained of a small amount of haemoptysis and on day 3 at 65 hours there was a significant deterioration in the clinical condition of the patient. Her vital signs included a temperature of $36^{\circ} \mathrm{C}$, blood pressure $130 / 80$, respiratory rate of 39 breaths per minute, heart rate of 90 and oxygen saturation of $40 \%$ on room air. Dyspnoea and haemoptysis were not associated with chest pain and symptoms were somewhat relieved by the upright position, not recumbent. This was improved to $92 \%$ with facial mask oxygen at a flow rate of $10 \mathrm{~L} /$ minute. Malaria smear was negative with only $P$. vivax gametocytes detected (Table 1 ). A complete blood count showed a haemoglobin of $7.2 \mathrm{mg} / \mathrm{dL}$, HCT $22.6 \%$, white blood cell count $5.5 \times 10^{3} / \mathrm{ml}$ with a differential counts $\left(\times 10^{3} / \mathrm{ml}\right)$ of: neutrophils (4.4), lymphocytes (0.8), monocytes (0.3), eosinophils (0) and basophils (0), and platelet count of $178 \times 10^{3} / \mu \mathrm{L}$. The $\mathrm{C}$-reactive protein was $80.8 \mathrm{mg} / \mathrm{L}$, sodium $131.7 \mathrm{mEq} / \mathrm{L}$, potassium $3.58 \mathrm{mEq} / \mathrm{L}$, urea nitrogen 10.2 units, creatinine $1.0 \mathrm{mg} / \mathrm{dL}$, total bilirubin $1.58 \mathrm{mg} / \mathrm{dL}$, direct bilirubin $0.74 \mathrm{mg} / \mathrm{dL}$, AST $67 \mathrm{U} / \mathrm{L}$, ALT $22 \mathrm{U} / \mathrm{L}$, alkaline phosphatase $588 \mathrm{u} / \mathrm{L}$, total protein $6.5 \mathrm{~g} / \mathrm{dL}$, and anion gap $15.2 \mathrm{mmol} / \mathrm{L}$, all of which were considered to be consistent with recovering acute malaria.

Intensive care was not available at the field hospital where the patient had been admitted and the patient was referred to the district hospital ( 75 minutes by road) where she was intubated in the casualty department and admitted to the intensive care unit. Arterial blood gases obtained after intubation with $100 \%$ oxygen revealed a $\mathrm{pH}$ of 7.32, $\mathrm{PaCo} 234 \mathrm{mmHg}$, a base excess $-8.7 \mathrm{mmol} / \mathrm{L}$, and

Table 1 Parasite clearance with fixed mefloquine-artesunate once daily dosing

\begin{tabular}{lccc}
\hline Day & & \multicolumn{2}{c}{ Parasitaemia/uL } \\
\cline { 3 - 4 } (hour) & Trophozoites & Schizonts & Gametocytes \\
\hline $0(0)$ & P. vivax 5/1,000 RBC & P. vivax 55/500 WBC & P. vivax $10 / 500$ WBC \\
$1(21)$ & P. vivax 4/500 WBC & P. vivax $1 / 500$ WBC & P. vivax $75 / 500$ WBC \\
$2(48)$ & Negative & Negative & P. vivax 3/500 WBC \\
$3(65)$ & Negative & Negative & P. vivax $1 / 500$ WBC \\
\hline
\end{tabular}

na: not applicable as calculated for trophozoite only. 
$\mathrm{SaO} 2$ 87\%. Electrocardiogram was normal as was an echocardiogram which showed normal right-sided heart pressures and volumes, and a chest radiograph showed bilateral pulmonary (fluffy) infiltrates. Two separate sputum tests for acid fast bacilli were negative. Treatment with artesunate, clindamycin, chloramphenicol, ceftriaxone, and frusemide was commenced. An induction on the second day of hospitalization (day 4 since first diagnosis of $P$. vivax) for known fetal death in utero resulted in a forceps delivery of a stillborn 2,500 g normal male infant. Unfortunately, after delivery ventilator pressures continued to increase [9]. On day 5 her condition worsened with bilateral pneumothorax. A CT scan with contrast done after insertion of bilateral chest drains reported ground glass opacity of the lung tissue and no pleural effusion. The patient had a cardiac arrest later the same day and died on day 7. No autopsy was done.

\section{Species PCR}

Nested PCR was performed on DNA extracted from 565 $\mathrm{uL}$ of packed red blood cells obtained just prior to treatment, following the protocol [10] with the following exceptions: the QIAamp DNA blood mini kit (Qiagen, Germany) was used to extract the DNA and Bioline reagents (BIOTAQ ${ }^{\mathrm{Tm}}$ DNA polymerase) were used to set up $20 \mu \mathrm{l} \mathrm{PCR}$ reactions. The sensitivity of the nested PCR assay at SMRU is 1 parasite/uL of whole blood for $P$. falciparum; 1 parasite/uL of whole blood for $P$. vivax; 10 parasites/uL of whole blood for Plasmodium malariae and 6.3 parasites/uL of whole blood for Plasmodium ovale. Using these methods the sample was found to be positive only for P. vivax and negative for other Plasmodium spp.

\section{Acute respiratory distress syndrome, malaria and pregnancy}

While sequestration of $P$. vivax in the lung circulation has been suggested clinically [4] but not proven $[6,11]$ there are in vitro conditions where adherence of $P$. vivax-infected red blood cells (RBC) has been demonstrated (immobilized chondroitin sulphate $\mathrm{A}$ and hyaluronic acid and fresh placental cells [12]; chondroitin sulphate A and intracellular adhesion molecule-1 receptors on human lung endothelial cells, Saimiri brain endothelial cells and placental cryosections [13]) although to a lesser extent than observed with P. falciparum. In non-pregnant adults, P. vivax-associated ARDS typically occurs after parasitaemia has declined on day 2 or 3 and a review of published case reports indicate that rapid intubation and ventilation are important for survival and cases that have survived were not pregnant $(91.7 \%(22 / 24))[5,14]$. In a broad review of the clinical spectrum or $P$. vivax, ARDS as a single complication has been reported in low numbers over the last 20 years, mainly from high income, nonendemic countries with a low case fatality rate. This is contrary to much higher rates (case fatality rate of 50$67 \%$ ) from more recent reports from endemic countries of severe vivax with ARDS occurring as part of multiple organ dysfunction/failure [15]. The risk of ARDS in relation to uncomplicated $P$. vivax has not been quantified, while for P. falciparum it has been estimated to be $0.1 \%$ (three in 3,300) US Army soldiers in Vietnam [16].

Systematic malaria screening (and treatment when positive) of all pregnant women at weekly antenatal consultations was introduced in 1986 at SMRU to reduce $P$. falciparum malaria-related maternal mortality [3] which was estimated at $1 \%$ of all pregnant women annually [2]. Since then and until the patient in this case died, there were 48,983 prospectively followed pregnancies with a known outcome (birth or miscarriage) and 12 P. falciparum and one $P$.vivax (the case in point) related maternal deaths, with each species accounting for one ARDS death. Amongst this cohort of pregnant women, malaria screening detected at least one episode of $P$. falciparum or $P$. vivax in 4,158 and 4,298 women, respectively. Assuming the case in point was caused by $P$. vivax the comparative rates of malaria-related mortality were: $P$. falciparum 2.89 per $1,000(12 / 4,158)$ and $P$. vivax 0.23 per $1,000(1 / 4,298)$, $\mathrm{P}=0.003$; whereas the ARDS-related malaria mortality was not significantly different for $P$. falciparum 0.24 per $1,000(1 / 4,158)$ and for $P$. vivax and 0.23 per $1,000(1 /$ 4,298) $\mathrm{P}=1.000$.

Reviews of cases suggest $P$. vivax-associated ARDS is mostly reported to occur after the start of anti-malarial treatment and potentially more cases will be seen with the spread of chloroquine-resistance and use of alternative regimens without the same anti-inflammatory drug properties [15]. However, mefloquine, which was the partner to artesunate in this case, also has anti-inflammatory properties [17].

\section{Conclusion}

The maternal death reported here is highly unusual in the context of the very high numbers of prospectively followed pregnancies with a confirmed diagnosis and treatment of $P$. vivax in this population. The patient was in late pregnancy, symptomatic, moderately anaemic with known $\beta$-thalassaemia trait and with a relatively high $P$. vivax parasitaemia. She was treated for uncomplicated malaria infection, took oral artemisinin-based anti-malarials and appeared to be recovering well. Unexpectedly ARDS developed on day 3 after treatment was completed and when trophozoites were no longer detectable on the peripheral blood smear. Although ventilated, she died from complications of ventilation. While no other cause for ARDS was diagnosed in this patient, she did have a low body weight and without autopsy an underlying condition cannot be completely ruled out. While most $P$. vivax-associated ARDS cases reported in the literature have recovered, they 
were not pregnant. This case of ARDS-associated mortality may have been related to the original $P$. vivax infection but this cannot be proven. If it is assumed that the patient's cause of death was caused by $P$. vivax-associated ARDS, then the risk of ARDS-related maternal mortality in this setting was similar for $P$. falciparum and $P$. vivax infection, contrary to malaria-related mortality which was more than 12-fold higher with P. falciparum.

\section{Consent}

Written informed consent was obtained from the patient for publication before she died as she was enrolled to a study (http://clinicaltrials.gov/ct2/show/NCT01054248) approved by the Oxford Tropical Research Ethics Committee (reference 45-09) and the Faculty of Tropical Medicine, Mahidol University, Thailand (reference MUTM 2009-06501). A copy of the written consent is available for review by the Editor-in-Chief of this journal.

\section{Abbreviations}

ANC: Antenatal clinic; ARDS: Acute respiratory distress syndrome;

HCT: Haematocrit; HIV: Human immunodeficiency virus; SMRU: Shoklo malaria research unit.

\section{Competing interests}

The authors declare they have no financial competing interests to declare. One of the authors (RM) is on the editorial board of Malaria Journal.

\section{Authors' contributions}

CC and NWT managed the patient. KW contributed to the laboratory work. RM, NJW and FN conceived of the particular study to which the patient was enrolled. RM, KW, CC and NWT prepared the first draft of the manuscript. All authors were involved in the revision of the draft manuscript and have read and approved the final manuscript.

\section{Acknowledgements}

We would like to acknowledge the contribution of the SMRU field staff and particularly the care from the Mae Sot Hospital staff. The study is part of SMRU and MORU, both part of the Thailand Major Overseas Program supported by the Wellcome Trust (UK).

\section{Author details}

'Shoklo Malaria Research Unit, Mahidol-Oxford Tropical Medicine Research Unit, Faculty of Tropical Medicine, Mahidol University, Mae Sot, Thailand. ${ }^{2}$ Mahidol-Oxford Tropical Medicine Research Unit, Faculty of Tropical Medicine, Mahidol University, Bangkok, Thailand. ${ }^{3}$ Centre for Tropical Medicine, Nuffield Department of Medicine, University of Oxford, Oxford, UK.

Received: 3 April 2014 Accepted: 22 May 2014

Published: 27 May 2014

\section{References}

1. Nosten F, ter Kuile F, Maelankirri L, Decludt B, White NJ: Malaria during pregnancy in an area of unstable endemicity. Trans $R$ Soc Trop Med Hyg 1991, 85:424-429.

2. Nosten F, McGready R, Simpson JA, Thwai KL, Balkan S, Cho T, Hkirijaroen L, Looareesuwan S, White NJ: Effects of Plasmodium vivax malaria in pregnancy. Lancet 1999, 354:546-549.

3. McGready R, Boel M, Rijken MJ, Ashley EA, Cho T, Moo O, Paw MK Pimanpanarak M, Hkirijareon L, Carrara VI, Lwin KM, Phyo AP, Turner C, Chu CS, van Vugt M, Price RN, Luxemburger C, ter Kuile FO, Tan SO, Proux S, Singhasivanon P, White NJ, Nosten FH: Effect of early detection and treatment on malaria related maternal mortality on the north-Western border of Thailand 1986-2010. PLoS One 2012, 7:e40244.

4. Anstey NM, Jacups SP, Cain T, Pearson T, Ziesing PJ, Fisher DA, Currie BJ, Marks PJ, Maguire GP: Pulmonary manifestations of uncomplicated falciparum and vivax malaria: cough, small airways obstruction, impaired gas transfer, and increased pulmonary phagocytic activity. $J$ Infect Dis 2002, 185:1326-1334.

5. Tan LK, Yacoub S, Scott S, Bhagani S, Jacobs M: Acute lung injury and other serious complications of Plasmodium vivax malaria. Lancet Infect Dis 2008, 8:449-454.

6. Valecha N, Pinto RG, Turner GD, Kumar A, Rodrigues S, Dubhashi NG, Rodrigues E, Banaulikar SS, Singh R, Dash AP, Baird JK: Histopathology of fatal respiratory distress caused by Plasmodium vivax malaria. Am J Trop Med Hyg 2009, 81:758-762.

7. Rijken MJ, Boel ME, Russell B, Imwong M, Leimanis ML, Phyo AP, Muehlenbachs A, Lindegardh N, McGready R, Rénia L, Snounou G, Singhasivanon P, Nosten F: Chloroquine resistant vivax malaria in a pregnant woman on the western border of Thailand. Malar J 2011, 10:113.

8. University of Oxford. In Randomised Trial of 3 Artemisinin Combination Therapy for Malaria in Pregnancy (DMA); 2010. accessed 01-Mar-2014. http://clinicaltrials.gov/ct2/show/NCT01054248.

9. Neligan PJ, Laffey JG: Clinical review: special populations-critical illness and pregnancy. Crit Care 2011, 15:227.

10. Singh B, Bobogare A, Cox-Singh J, Snounou G, Abdullah MS, Rahman HA: A genus- and species-specific nested polymerase chain reaction malaria detection assay for epidemiologic studies. Am J Trop Med Hyg 1999, 60:687-692.

11. Taylor WR, Hanson J, Turner GD, White NJ, Dondorp AM: Respiratory manifestations of malaria. Chest 2012, 142:492-505.

12. Chotivanich K, Udomsangpetch R, Suwanarusk R, Pukrittayakamee S, Wilairatana P, Beeson JG, Day NP, White NJ: Plasmodium vivax adherence to placental glycosaminoglycans. PLoS One 2012, 7:e34509.

13. Carvalho BO, Lopes SC, Nogueira PA, Orlandi PP, Bargieri DY, Blanco YC, Mamoni R, Leite JA, Rodrigues MM, Soares IS, Oliveira TR, Wunderlich G, Lacerda MV, del Portillo HA, Araújo MO, Russell B, Suwanarusk R, Snounou G, Rénia L, Costa FT: On the cytoadhesion of Plasmodium vivax-infected erythrocytes. J Infect Dis 2010, 202:638-647.

14. Sarkar S, Saha K, Das CS: Three cases of ARDS: An emerging complication of Plasmodium vivax malaria. Lung India 2010, 27:154-157.

15. Anstey NM, Douglas NM, Poespoprodjo JR, Price RN: Plasmodium vivax: clinical spectrum, risk factors and pathogenesis. Adv Parasitol 2012, 80:151-201

16. Sheehy TW, Reba RC: Complications of falciparum malaria and their treatment. Ann Intern Med 1967, 66:807-809.

17. Jaroensuk J, Stoesser N, Leimanis ML, Jittamala P, White NJ, Nosten FH, McGready R: Treatment of suspected hyper-reactive malarial splenomegaly (HMS) in pregnancy with mefloquine. Am J Trop Med Hyg 2014, 90:609-611.

doi:10.1186/1475-2875-13-191

Cite this article as: McGready et al:: Uncomplicated Plasmodium vivax malaria in pregnancy associated with mortality from acute respiratory distress syndrome. Malaria Journal 2014 13:191.

\section{Submit your next manuscript to BioMed Central and take full advantage of:}

- Convenient online submission

- Thorough peer review

- No space constraints or color figure charges

- Immediate publication on acceptance

- Inclusion in PubMed, CAS, Scopus and Google Scholar

- Research which is freely available for redistribution 